\title{
Three-dimensional trajectory optimization for multi-stage launch vehicle mission using a full-space quasi-Lagrange-Newton method
} $\begin{array}{cc}\text { Hsuan-Hao Wang }^{1} & \text { Yi-Su Lo } \\ \text { Feng-Nan Hwang } & \\ & \text { Feng-Tai Hwang }^{3}\end{array}$

(Received 28 February 2019; revised 14 June 2019)

\begin{abstract}
Many aerospace industrial applications require robust and efficient numerical solutions of large sparse nonlinear constrained parameter optimization problems arising from optimal trajectory problems. A three-dimensional multistage launcher problem is taken as a numerical example for studying the performance and applicability of the full-space Lagrange-Newton-Krylov method. The typical optimal trajectory, control history and other important physical qualities are presented, and the efficiency of the algorithm is also investigated.
\end{abstract}

DoI:10.21914/anziamj.v60i0.14067 gives this article, (c) Austral. Mathematical Soc. 2019. Published August 30, 2019, as part of the Proceedings of the 18th Biennial Computational Techniques and Applications Conference. ISSN 1445-8810. (Print two pages per sheet of paper.) Copies of this article must not be made otherwise available on the internet; instead link directly to the DOI for this article. 


\section{Contents}

1 Introduction

C173

2 A full-space optimization solution algorithm for launch vehicle problems

C174

3 Numerical results and discussions

C179

\section{Introduction}

Space missions are expensive. To conduct a cost-effective space mission, 'optimization' is an essential task in many aspects of the mission life. The optimal control method is one of the optimization techniques commonly used in space engineering with a broad range of applications, such as low-thrust spacecraft orbit transfer problems and launcher optimal ascending trajectory design problems [3]. The primary goal of a multistage rocket trajectory design is to find a trajectory from the launch site to satellite insertion point which minimizes the launcher's total energy or other performance indices. The general procedure for solving this optimization problem is to recast a continuous time optimal trajectory problem as a large, sparse algebraic constrained parameter optimization problem by discretizing the dynamic constraints with some numerical integrator scheme, such as the Euler method, classical Runge-Kutta method, trapezoidal method or Hermite-Simpson method [1].

Wang et al. [5] proposed the full-space quasi Lagrange-Newton-Krylov (FQLNK) method for solving the resulting parameter optimization problem and presented a two-dimensional multistage launch vehicle example. In this approach, the candidate optimal solution is found through solving the first-order necessary condition, also known as the Karush-Kuhn-Tucker (KKT) condition [1], with an inexact Newton method by introducing the Lagrangian function into 
the objective function. At each Newton iteration, the KKT system is solved for finding a search direction by a Krylov-subspace method in conjunction with an incomplete LU preconditioner. The numerical experiments showed the most expensive part of the overall computation is in constructing the subblock of the KKT matrix corresponding to the Hessian matrix of the Lagrangian function, where the Broyden-Fletcher-Goldfarb-Shanno (BFGS) formula was employed as an approximation scheme to reduce the computational cost. A superior speedup of the BFGS-based FQLNK algorithm was demonstrated compared to some of the alternatives, including finite differences and automatic differentiation.

To obtain a trajectory close to a realistic situation, we consider the threedimensional mathematical model of a multistage trajectory optimization problem by taking the Coriolis force into account. In the next section we build the mathematical optimal control model for a multistage launch vehicle system as a parameter optimization problem. In Section 3 we present some numerical results and discussions.

\section{A full-space optimization solution algorithm for launch vehicle problems}

We consider a three-stage launch vehicle which is used to deliver a microsatellite with mass ranging from 70 to $105 \mathrm{~kg}$ into a low-Earth circular orbit with the target altitude between 400 to $630 \mathrm{~km}$. The system engineer is interested in finding an optimal trajectory for the launch vehicle with a minimum flight duration to ensure that the telemetry, tracking, and control system works properly between the ground station and the launch vehicle. The launch azimuth angle is set to $196^{\circ}$, the longitude and latitude of the launch site are $120.89^{\circ}$ and $22.14^{\circ}$, respectively. Table 1 summarizes the structure and propulsion data for each stage of the launch vehicle, and Table 2 lists the flight sequence of the three-stage launch vehicle. The fairing, which is utilized 
Table 1: The structure, mass distribution and thrust for each stage of the three-stage launch vehicle.

\begin{tabular}{lrrr} 
Stage & I & II & III \\
\hline Reference area $\left(\mathrm{m}^{2}\right)$ & 0.7854 & 0.7854 & 0.1564 \\
Motor mass $(\mathrm{kg})$ & 10091 & 1906 & 344 \\
Propellant mass $(\mathrm{kg})$ & 8880 & 1677 & 296 \\
Thrust (N) & 243824 & 57555.4 & 6085.8 \\
Burn time (s) & 100 & 80 & 133
\end{tabular}

Table 2: The flight sequence of the three-stage launch vehicle, where $\Delta t_{c}$ is the time of the coasting flight.

\begin{tabular}{ll} 
Time $(\mathrm{s})$ & Events \\
\hline$t_{0}=0$ & Stage I ignition and lift-off \\
$t_{1}=5$ & Beginning of kick-turn (pitch down) \\
$t_{2}=100$ & Stage I burnout, stages I and II separation, \\
& and stage II ignition \\
$t_{3}=180$ & Stage II burnout, stages II and III separation, \\
& and beginning of the coasting flight \\
$t_{4}=180+\Delta t_{c}$ & End of the coasting flight, and stage III ignition \\
$t_{5}=313+\Delta t_{c}$ & Stage III burnout and orbit insertion
\end{tabular}

not only to reduce the effect of air resistance but also to protect the payload (satellite) from contamination by the atmosphere, is set to be jettisoned when the launch vehicle reaches the altitude of $100 \mathrm{~km}$.

To build a mathematical model for the minimum time trajectory design problem of a multistage launch vehicle with some coasting-flight period, we first put forward the following assumptions.

(1) The Earth is perfectly spherical, so the latitude is the geocentric latitude, and the normal vector at any point of the surface is a vector passing through the centre of the Earth. 


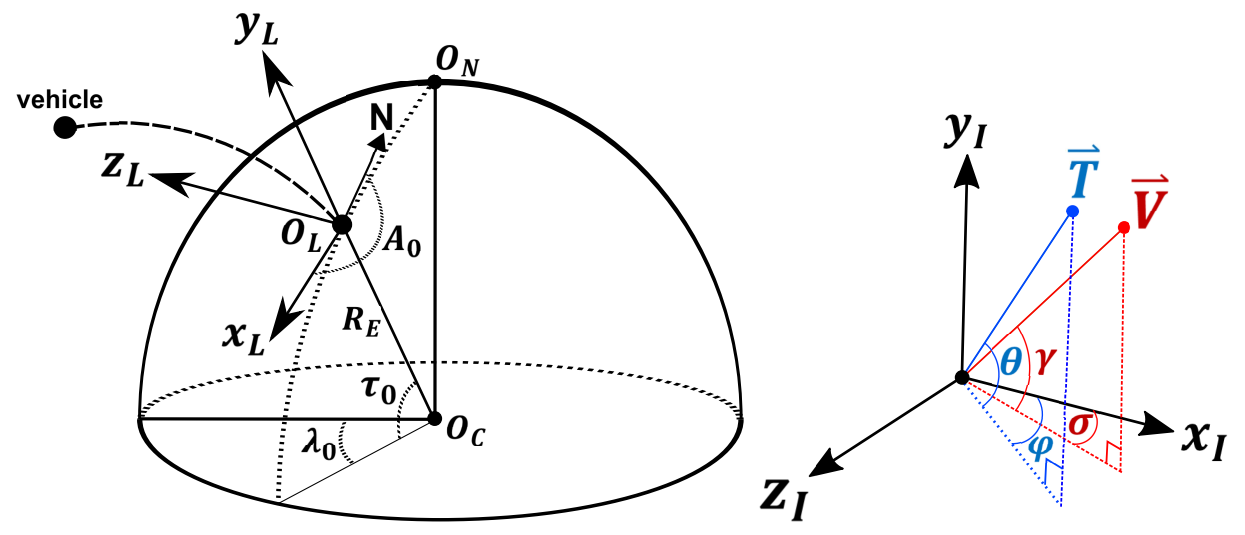

Figure 1: Angle definition and control variables.

(2) For aerodynamics, we do not take the impact of either the wind or the lift force into accounts; only air resistance is considered.

(3) The mass distribution, burn time, and thrusts for each stage of the launch vehicle are available. The thrust is stable, and the mass of the fuel is a linearly decreasing function of the burn time.

(4) During the flight, the vehicle is regarded as a particle, so its vehicle attitude is ignored.

(5) To avoid performing any coordinate conversion, we set the fixed launch point inertial (LPI) frame (see the left of Figure 1) as the coordinate reference system.

Figure 1 defines some associate vectors and angles. The thrust vector is $\vec{T}$ and $\vec{V}$ is the velocity vector. The two controllable angles are the pitch angle $\theta$ and the yaw angle $\varphi$. Furthermore, the two flight angles are the flight path angle $\gamma$ and the side slip angle $\sigma$.

The multistage rocket launch process starts at time $t_{0}$, ends at time $t_{f}$, and consist of six events over times $t_{0}<t_{1}<t_{2}<\cdots<t_{5}=t_{f}$. The launch vehicle under consideration involves more than one stage and possibly complex 
mission sequence. Some of the state or other variables may be discontinuous at particular time points, which are referred to as events. The semi-closed time interval $\left[t_{i-1}, t_{i}\right)$ is called the $i$ th phase, where $i=1, \ldots, 5$. The time period of the $i$ th phase is defined as $\Delta t_{i}=t_{i}-t_{i-1}$. The one coasting period lasts for $\Delta t_{c}$ during the fourth phase, $\left[t_{3}, t_{4}\right]$.

The launch vehicle trajectory design problem is formulated as a free final time optimal control problem as follows. Find two piecewise continuous functions, pitch angle $\theta(t)$ and yaw angle $\varphi(t)$, on the time interval $\left[t_{0}, t_{f}\right]$ that minimize the objective function $\mathrm{J}=\mathrm{t}_{\mathrm{f}}$ subject to the dynamic constraints

$$
\frac{d s}{d t}=f^{(i)}[s(t), c(t)] \quad \text { with } t \in\left[t_{i-1}, t_{i}\right] \quad \text { for } i=1, \ldots, 5,
$$

and the initial and final state conditions $\psi_{0}\left[s\left(t_{0}\right)\right]=0$ and $\psi_{f}\left[s\left(t_{f}\right)\right]=0$, respectively. Here, the vector $s=(\mathfrak{u}, v, w, x, y, z)^{\top}$ is the set of the state variables, $c=(\theta, \varphi)^{\top}$ is the set of the control variables, and $\vec{V}=(u, v, w)$ is the velocity of the rocket at position $(x, y, z)$. The final time $t_{f}$, which is referred to a design variable, is to be determined.

The dynamic constraints are explicitly defined as

$$
f^{(i)}[s(t), c(t)]=\left(\begin{array}{c}
\frac{T^{(i)}}{M^{(i)}} \cos \theta \cos \varphi-\frac{D}{M^{(i)}} \cos \gamma \cos \sigma-g \frac{x}{\|r\|}+a_{c x} \\
\frac{T^{(i)}}{M^{(i)}} \sin \theta \cos \varphi-\frac{D}{M^{(i)}} \sin \gamma \cos \sigma-g \frac{y+R_{E}}{\|r\|}+a_{c y} \\
-\frac{T^{(i)}}{M^{(i)}} \sin \varphi+\frac{D}{M^{(i)}} \sin \sigma-g \frac{z}{\|r\|}+a_{c z} \\
u \\
v
\end{array}\right),
$$

for $i=1, \ldots, 5$ and where $T^{(i)}$ is the thrust, $M^{(i)}$ is the total mass at the ith phase, including the structure mass and the fuel mass, and $\|\vec{r}\|$, with $\vec{r}=\left(x, y+R_{E}, z\right)$, is the distance between the rocket and the centre of the earth. Here $R_{E}$ is average radius of the Earth. The flight path angle $\gamma$ and side slip angle $\sigma$ are defined as $\gamma=\tan ^{-1}(v / u)$ and $\sigma=-\sin ^{-1}(w / V)$, respectively. During the coasting-flight period the thrust is zero, $\mathrm{T}^{(4)}=0$, 
and the control variables $\theta$ and $\varphi$ are determined solely by the motion of the vehicle. Furthermore, the air resistance $D=\frac{1}{2} \rho V^{2} C_{D} S_{\text {ref }}$, where $C_{D}$ is the drag coefficient changing with Mach number and $S_{\text {ref }}$ is the cross-sectional area of the vehicle, $\|\vec{V}\|=\sqrt{u^{2}+v^{2}+w^{2}}$ is the total velocity, the density of the air is $\rho=\rho_{0} \exp \left[\left(R_{E}-\|\vec{r}\|\right) / H\right]$, the density of air at sea level is $\rho_{0}$, the thickness of earth's atmosphere is $H$, and the gravity $g=g_{0}\left(R_{E} /\|\vec{r}\|\right)^{2}$ is the first order model. Here, $g_{0}$ is the gravity at sea level.

The Earth rotates counterclockwise, viewed from above the North pole; therefore the Coriolis force for objects moving high speed cannot be ignored. The Coriolis force on the LPI frame is

$$
\left(\begin{array}{l}
a_{c x} \\
a_{c y} \\
a_{c z}
\end{array}\right)=-2 \vec{\omega} \times \vec{V}=\left(\begin{array}{c}
2\left(\omega_{z} v-\omega_{y} w\right) \\
2\left(\omega_{x} w-\omega_{z} u\right) \\
2\left(\omega_{y} u-\omega_{x} v\right)
\end{array}\right),
$$

where the rotation vector

$$
\vec{\omega}=\left(\begin{array}{c}
\omega_{x} \\
\omega_{y} \\
\omega_{z}
\end{array}\right)=\Omega_{E}\left(\begin{array}{c}
\cos \tau_{0} \cos A_{0} \\
\sin \tau_{0} \\
-\cos \tau_{0} \sin A_{0}
\end{array}\right) .
$$

Here, the spin rate of the Earth $\Omega_{\mathrm{E}}$ is $2 \pi / 86400 \approx 7.2722 \times 10^{-5} \mathrm{rad} / \mathrm{s}$, the launch azimuth is $A_{0}=196^{\circ}$ and geocentric latitude of the launch point is $\tau_{0}=22.14^{\circ}$.

The initial condition is prescribed as

$$
\psi_{0}\left[s\left(t_{0}\right)\right]=\left(\begin{array}{l}
\left\|\vec{r}\left(t_{0}\right)\right\|-r_{0} \\
\left\|\vec{V}\left(t_{0}\right)\right\|-V_{0}
\end{array}\right)=0
$$

and the final condition is prescribed as

$$
\psi_{f}\left[s\left(t_{f}\right)\right]=\left(\begin{array}{c}
\left\|\vec{r}\left(t_{f}\right)\right\|-R_{E}-H\left(t_{f}\right) \\
\left\|V\left(\overrightarrow{t_{f}}\right)\right\|-\sqrt{\mu /\left\|\vec{r}\left(t_{f}\right)\right\|} \\
\left(\vec{r}\left(t_{f}\right) \cdot \vec{V}\left(t_{f}\right)\right) /\left(\left\|\vec{r}\left(t_{f}\right)\right\|\left\|\vec{V}\left(t_{f}\right)\right\|\right)
\end{array}\right)=0 .
$$


Condition (3) is an insertion condition to assure that the launch vehicle reaches enough height, $\mathrm{H}\left(\mathrm{t}_{\mathrm{f}}\right)$, and sufficient speed, $\sqrt{\mu /\left\|\vec{r}\left(t_{\mathrm{f}}\right)\right\|}$, with an appropriate angle. Here, $\mu$ is the gravitational parameter of the Earth.

All state parameters are assumed to be continuous at each $t_{i}$; hence, the linkage condition between each stage is imposed, $s\left(t_{i}^{-}\right)=s\left(t_{i}^{+}\right)$for $i=1,2,3,4$. Here, $s\left(t_{i}^{-}\right) \equiv \lim _{t \leftarrow t_{i}^{-}} s(t)$ and $s\left(t_{i}^{+}\right) \equiv \lim _{t \leftarrow t_{i}^{+}} s(t)$ are the left-hand and righthand limits of $s$ at $t=t_{i}$, respectively.

After a change of variable by introducing some pseudo-time variable and the discretization of the dynamic constraints (1) by using the trapezoidal rule [1], we finally derive a large sparse algebraic constrained parameter optimization from the continuous free final time control problem: Find the parameter vector $p_{x}$ that minimizes the objective function $J=\Delta t_{c}$ subject to the constraints $c\left(p_{x}\right)=0$. Here, $p_{x}$ is a vector of discrete state and control variables on each time grid point. This ODE-constrained optimization problem is then solved by the FQLNK algorithm. Define the Lagrangian functional $\mathcal{L}\left(p_{x}, p_{\lambda}\right) \equiv J\left(p_{x}\right)-\lambda^{\top} c\left(p_{x}\right)$, where $\lambda$ is a vector of Lagrangian multipliers. Figure 2 displays a flowchart of the algorithm for finding the critical solution of the KKT condition, $\nabla \mathcal{L}\left(p_{x}, p_{\lambda}\right)=\nabla \mathcal{L}(p)=0$ and we refer to Wang et al. [5] for a detailed description of each component of the FQLNK algorithm.

\section{$3 \quad$ Numerical results and discussions}

Numerical results are obtained by using the trapezoidal method as a numerical integrator with the grid $\left(n_{1}, n_{2}, n_{3}, n_{4}, n_{5}\right)=(2,32,32,32,32)$, where $n_{i}$ is the total number of subintervals for the ith phase. The equidistant grid is used for each phase with time step size $h^{(i)}=\left(t_{i}-t_{i-1}\right) / n_{i}$. The payload is set to $100 \mathrm{~kg}$, and the target altitude is $500 \mathrm{~km}$. The three-stage launch vehicle achieves the optimal orbit insertion point at $407.25 \mathrm{~s}$, including a coasting-flight period of $94.25 \mathrm{~s}$. Figure 3 shows the histories of the optimal controlled pitch angle and yaw angle, and the corresponding state variables: 


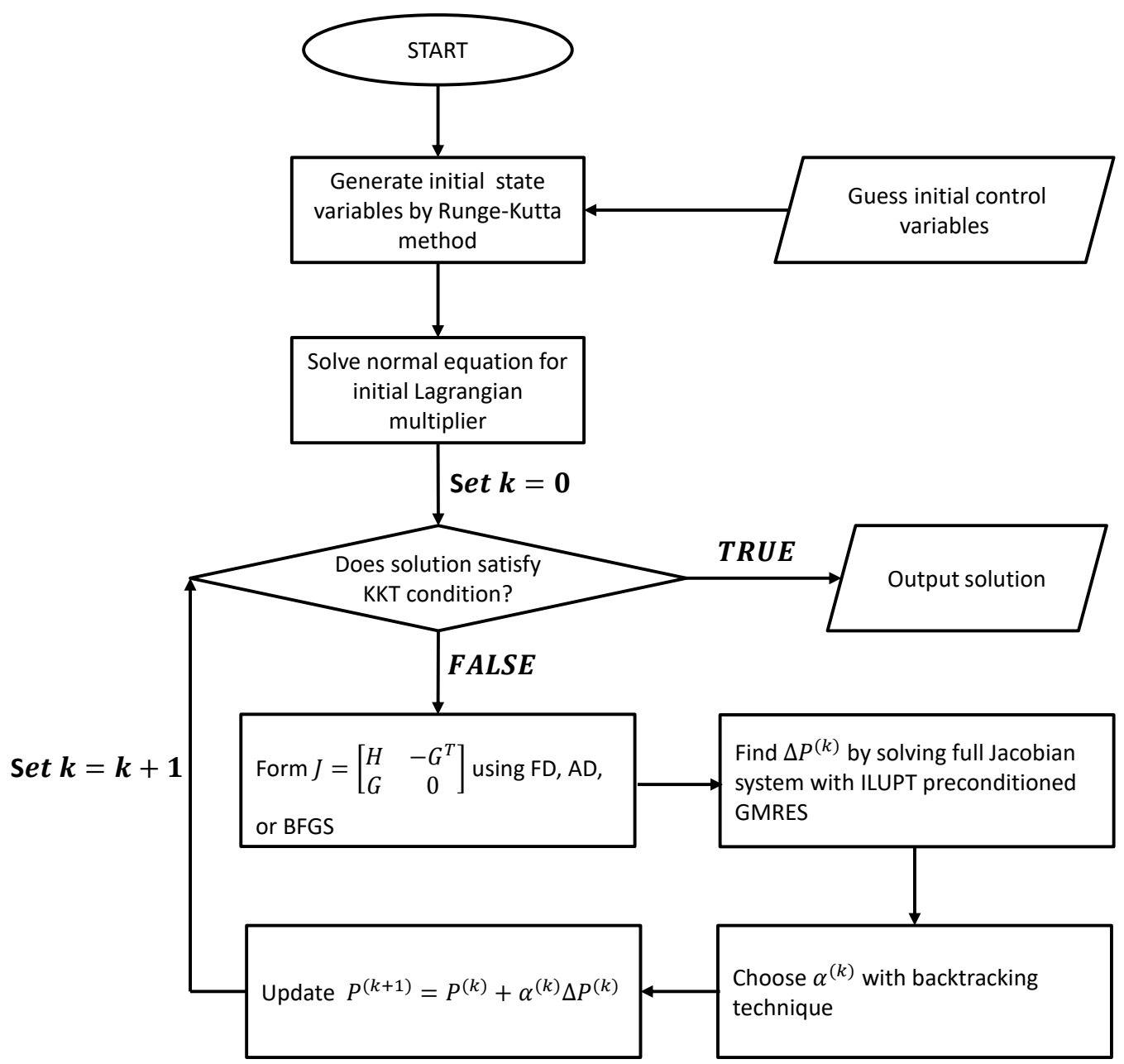

Figure 2: A flowchart of the full-space quasi-Lagrange-Newton-Krylov algorithm, including the initial guess construction [5]. 
the velocity and altitude. It is observed from the bottom-left of Figure 3, the velocity of the launch vehicle increases over time, except during the coasting-flight. At the end of the coasting flight and the start of the third stage ignition, the velocity decreases mainly because of gravity effects and no thrust during that period. In contrast, the altitude in the bottom-right of Figure 3 always increases. Figure 3 also suggests that a too long or too short coasting-flight period will cause the failure of the space mission since the target altitude cannot be reached.

The left of Figure 4 shows the history plot of the dynamic pressure and the angle of attack (AOA) during the entire space mission. The dynamic pressure for the launch vehicle is defined as $q=\frac{1}{2} \rho\|\vec{V}\|^{2}$, where $\rho$ is the air density and $\vec{V}$ is the velocity of the launch vehicle. Hence, we expect $q$ to be zero at the launch point and when the launch vehicle is outside the atmosphere, and non-negative otherwise. The maximum of the dynamic pressure $\max (\mathbf{q})$ is one of the most critical factors for the rocket launch process. When near $\max (\mathbf{q})$ the launch vehicle experiences the most significant aerodynamic structural load, and to prevent damage to the structure or to avoid losing control of the vehicle, we must make the AOA as close to zero degrees as possible. Figure 4 shows that the AOA corresponding to our optimal trajectory is at maintained at less than three degrees during the $\max (\mathbf{q})$ period. In this case, $\max (\mathbf{q})$ is at $t=40 \mathrm{~s}$ and around the altitude of $10 \mathrm{~km}$.

The right of Figure 4 displays the ground track plot, which is the projection of the flight trajectory onto the surface of the Earth. The ground track bends to the right because of changes in the yaw angle and the effect of Coriolis force.

The left of Figure 5 shows the altitude versus the downrange, which provides important information for rocket mission analysis or the setting of communication stations. The downrange is defined as the horizontal distance from the launch site to the launch vehicle. The right of Figure 5 illustrates how to calculate the downrange from the orbit of the launch vehicle where $\vec{r}_{0}=\left(x_{0}, y_{0}+R_{E}, z_{0}\right), \vec{r}_{1}=\left(x_{1}, y_{1}+R_{E}, z_{1}\right), \ldots, \vec{r}_{n}=\left(x_{n}, y_{n}+R_{E}, z_{n}\right)$ are the 

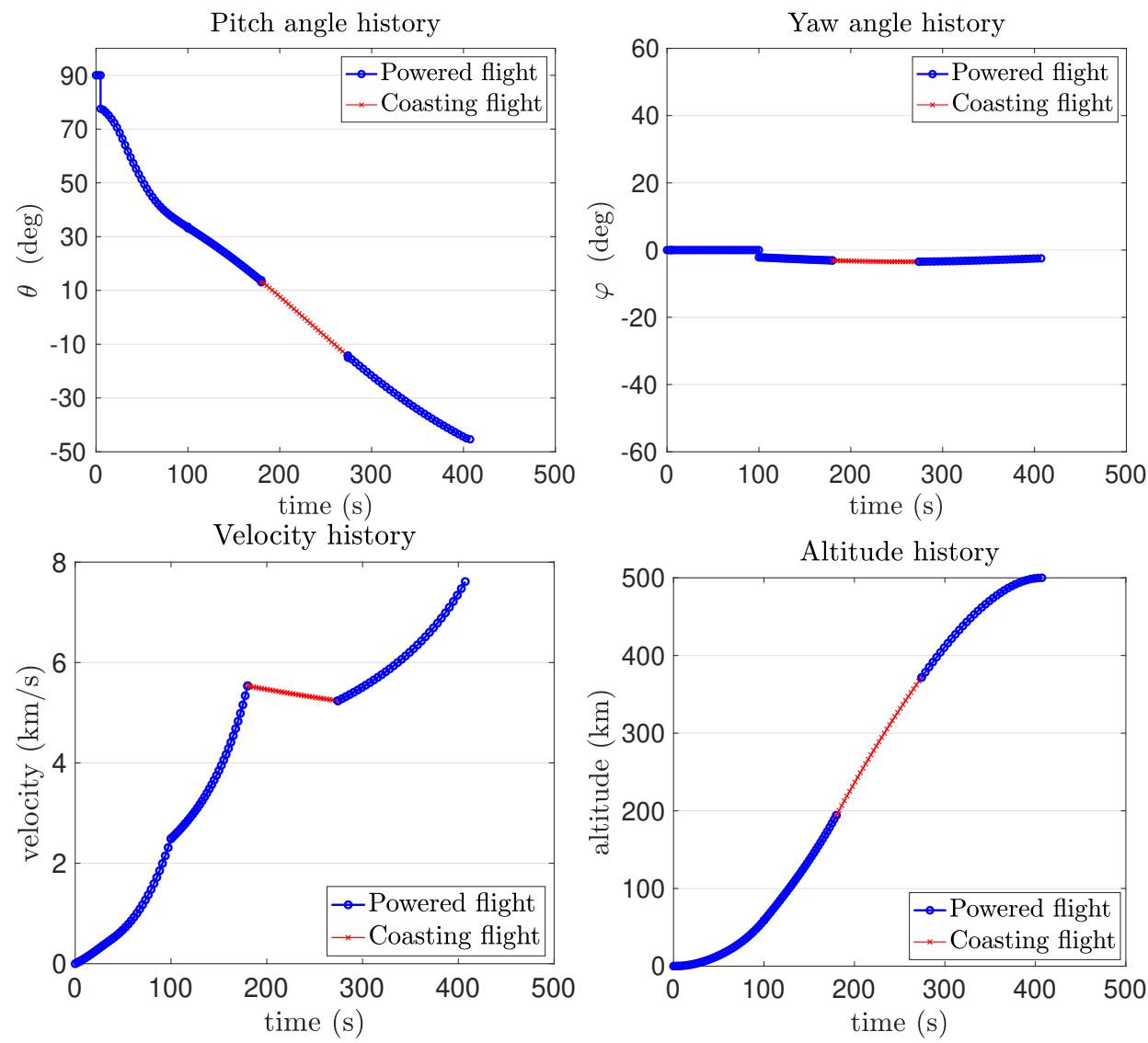

Figure 3: The optimal controlled pitch angle (top left), and yaw angle (top right) with the corresponding state variables: velocity (bottom left) and altitude (bottom right). 

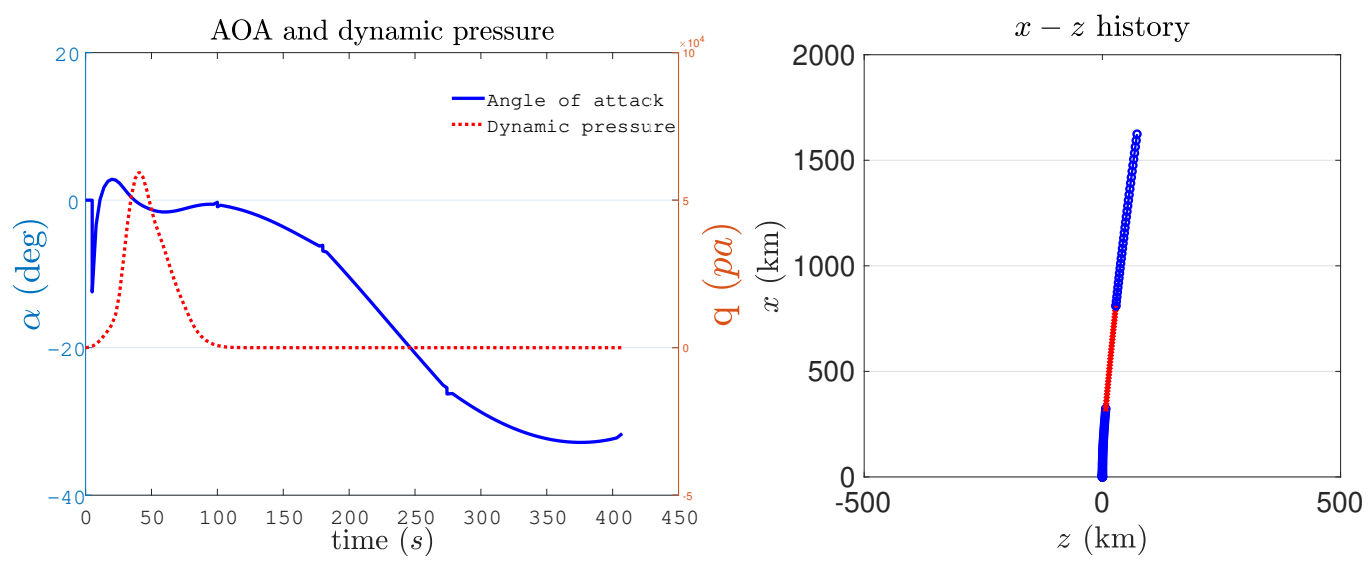

Figure 4: (Left) The history plot of dynamic pressure and angle of attack (AOA); (right) the ground track during the entire mission.

position vectors at each time step with respect to the centre of the Earth. The downrange $D_{\text {total }}=\sum_{i=1}^{n} D_{i}$ is computed by summing each arc length at each time step $D_{i}=R_{E} \cdot \phi_{i}$ with the range angle $\phi_{i}=\cos ^{-1}\left[\left(\vec{r}_{i-i} \cdot \vec{r}_{i}\right) /\left(\left\|\vec{r}_{i-i}\right\|\left\|\vec{r}_{i}\right\|\right)\right]$. Our mission is to make the coasting flight as short as possible which is equivalent to minimizing the downrange.

For comparison, we also consider a simplified two-dimensional model with zero yaw angle $(\varphi=0)$, zero side slip angle $(\sigma=0)$, and where the Coriolis force is ignored. The rocket structure parameters, flight sequence, initial and final conditions, and time grid are the same as for the 3D case. We use the optimal trajectory constrained by this $2 \mathrm{D}$ model as a guidance law for the numerical simulation of the launch mission and check whether the final satellite insertion condition is satisfied or not. Our numerical simulator uses a fourth-order explicit Runge-Kutta method for solving the 3D model (1). Table 3 summarizes the final conditions of the 2D and 3D cases. This table indicates that a 2D based guidance law can produce a sufficient insertion speed and the orthogonality condition is also satisfied. However, the altitude is not high enough and does not reach the required insertion point. One 


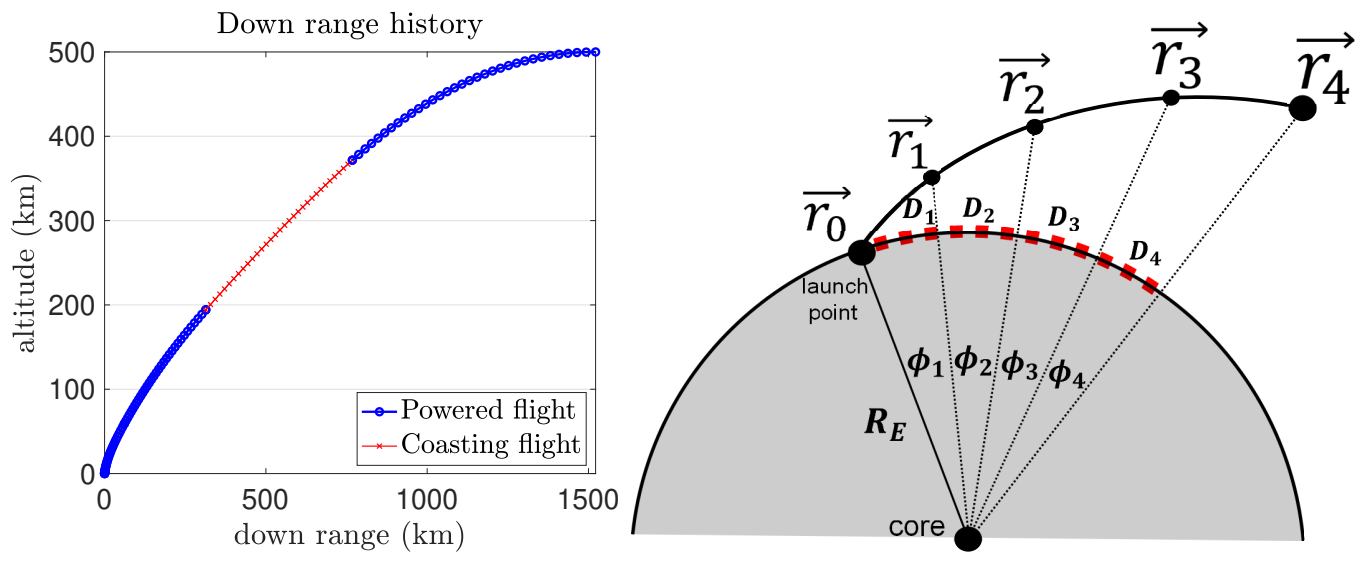

Figure 5: Downrange (left) and the downrange calculation (right).

Table 3: A comparison of the final conditions for the 2D and 3D models. final condition target $2 \mathrm{D}$ result $3 \mathrm{D}$ result

$\begin{array}{lrrr}\left\|\vec{r}\left(t_{f}\right)\right\|-R_{E}(\mathrm{~km}) & 500.00 & 492.23 & 500.47 \\ \left\|\vec{V}\left(t_{f}\right)\right\|(\mathrm{km} / \mathrm{s}) & 7.613 & 7.637 & 7.612 \\ \left.\cos ^{-1}\left[r\left(t_{f}\right) \cdot \vec{V}\left(t_{f}\right)\right) /\left(\left\|\vec{r}\left(t_{f}\right)\right\|\|\vec{V}\|\right)\right]^{\circ} & 90 & 90.41 & 89.99\end{array}$

key feature of our full-space method is that the optimal trajectory satisfies the final condition automatically, unlike the shooting based method. Hence, the three-dimensional model with Coriolis force is necessary for real space missions.

Table 4 shows the number of Newton iterations for different weights of the payload and different altitudes. We find that the FQLNK algorithm converges for all test cases. However, nonlinear iteration counts are sensitive to these system parameters. The same initial guess vector is used for all test cases and this vector is originally designed for a payload of $100 \mathrm{~kg}$ and a target altitude of $500 \mathrm{~km}$. Table 4 also suggests that the initial residual norm for the KKT condition can serve as an indicator of the degree of difficulty of the nonlinear problem. Roughly speaking, our solver needs more iterations for convergence 
Table 4: The numbers of Newton iterations, the coasting period, and the initial residual norm (in units of $\times 10^{-1}$ ) for different weights of the payload and different altitudes.

\begin{tabular}{lrrrrrr} 
Payload $(\mathrm{kg})$ & 70 & 80 & 90 & 100 & 105 & 120 \\
\hline$\Delta \mathrm{t}(\mathrm{s})$ & 37.3 & 53.5 & 71.4 & 92.9 & 105.0 & 119.6 \\
\# iterations & 28 & 17 & 10 & 8 & 16 & 25 \\
inital residual norm $\left(\times 10^{-1}\right)$ & 1.8 & 1.7 & 1.8 & 1.6 & 2.0 & 2.8
\end{tabular}

\begin{tabular}{lrrrrrr} 
Altitude $(\mathrm{km})$ & 400 & 450 & 500 & 550 & 600 & 630 \\
\hline$\Delta \mathrm{t}(\mathrm{s})$ & 36.0 & 63.4 & 92.9 & 96.5 & 151.6 & 170.2 \\
\# iterations & 28 & 5 & 8 & 8 & 10 & 13 \\
inital residual norm $\left(\times 10^{-1}\right)$ & 1.6 & 1.4 & 1.6 & 1.8 & 2.2 & 2.4
\end{tabular}

for the case with the larger initial residual norm. The enhancement of the robustness of FQLNK with nonlinear preconditioning techniques [6] with applications in trajectory optimization is the subject of an ongoing project. Other topics include generalization to a more realistic model, six degrees of freedom movement and attitude calculations [4], parallelization of the algorithm to accelerate the numerical simulation [6], and the extension of our solver to multi-objective optimization problems [2].

Acknowledgements This work was partially supported by the Ministry of Science and Technology of Taiwan, under Grant No. MOST-106-2115-M008-010-MY2.

\section{References}

[1] J. T. Betts. Practical methods for optimal control and estimation using nonlinear programming. 2nd. Advances in Design and Control. SIAM, 2010. DOI: $10.1137 / 1.9780898718577$ (cit. on pp. C173, C179). 
[2] R. T. Marler and J. S. Arora. "Survey of multi-objective optimization methods for engineering". In: Struct. Multidiscip. Opt. 26.6 (2004), pp. 369-395. DOI: 10.1007/s00158-003-0368-6 (cit. on p. C185).

[3] W. Roh and Y. Kim. "Trajectory optimization for a multi-stage launch vehicle using time finite element and direct collocation methods". In: Eng. Opt. 34 (2002), pp. 15-32. DOI: 10.1080/03052150210912 (cit. on p. C173).

[4] G. D. Silveira and V. Carrara. "A six degrees-of-freedom flight dynamics simulation tool of launch vehicles". In: J. Aero. Tech. Manag. 7 (2015), pp. 231-239. DOI: 10.5028/jatm.v7i2. 433 (cit. on p. C185).

[5] H.-H. Wang, Y.-S. Lo, F.-T. Hwang, and F.-N. Hwang. "A full-space quasi-Lagrange-Newton-Krylov algorithm for trajectory optimization problems". In: Electron. T. Numer. Anal. 49 (2018), pp. 103-125. DOI: 10.1553/etna_vol49s103 (cit. on pp. C173, C179, C180).

[6] H. Yang, F.-N. Hwang, and X-.C. Cai. "Nonlinear preconditioning techniques for full-space Lagrange-Newton solution of PDE-constrained optimization problems". In: SIAM J. Sci. Comput. 38 (2016), A2756-A2778. DOI: 10.1137/15M104075X (cit. on p. C185).

\section{Author addresses}

1. Hsuan-Hao Wang, Department of Mathematics, National Central University, Jhongli District, Taoyuan City 32001, Taiwan.

2. Yi-Su Lo, Department of Mathematics, National Central University, Jhongli District, Taoyuan City 32001, Taiwan.

3. Feng-Tai Hwang, Division of Satellite Image, National Space Organization, Hsinchu City 30078, Taiwan.

4. Feng-Nan Hwang, Department of Mathematics, National Central University, Jhongli District, Taoyuan City 32001, Taiwan. 